\title{
Homotopic Morphing of Planar Curves
}

\author{
N. Dym, A. Shtengel and Y. Lipman \\ Weizmann Institute of Science
}

\begin{abstract}
This paper presents an algorithm for morphing between closed, planar piecewise-C $C^{1}$ curves. The morph is guaranteed to be a regular homotopy, meaning that pinching will not occur in the intermediate curves.

The algorithm is based on a novel convex characterization of the space of regular closed curves and a suitable symmetric length-deviation energy. The intermediate curves constructed by the morphing algorithm are guaranteed to be regular due to the convexity and feasibility of the problem.

We show that our method compares favorably with standard curve morphing techniques, and that these methods sometimes fail to produce a regular homotopy, and as a result produce an undesirable morph.

We explore several applications and extensions of our approach, including morphing networks of curves with simple connectivity, morphing of curves with different turning numbers with minimal pinching, convex combination of several curves, and homotopic morphing of b-spline curves via their control polygon.
\end{abstract}

\section{Introduction}

Morphing is a classical problem in computer graphics. An animator supplies a source shape and a target shape, and the task is to automatically generate intermediate shapes, so as to create a natural and satisfying morph. The shapes supplied may be 3D or planar, and may or may not contain information on the interior of the shape. In this paper we consider the problem of morphing closed planar curves. The curves are assumed to be piecewise $C^{1}$, thus including $C^{1}$ curves and polygonal curves as special cases.

Morphing of curves is typically divided into two subproblems. The vertex correspondence problem deals with finding a correspondence between the given curves, while the vertex path problem deals with the construction of the intermediate curves using the supplied correspondence. We consider the second problem, and assume that the correspondence has been generated by a suitable algorithm, or supplied by the user.

A reasonable requirement of a morph is that all intermediate curves will be regular. A regular curve is a curve without extreme cusps. By this we mean a point on the curve where the tangent rotates by $180^{\circ}$, as shown in Figure 2 in (a)-middle for the polygonal case, (b)-middle for the $C^{1}$ case. Consequently, (a) and (b) show examples of nonregular morphs. The well-known Whitney-Graustein Theorem [Whi37] asserts that a morph which consists only of

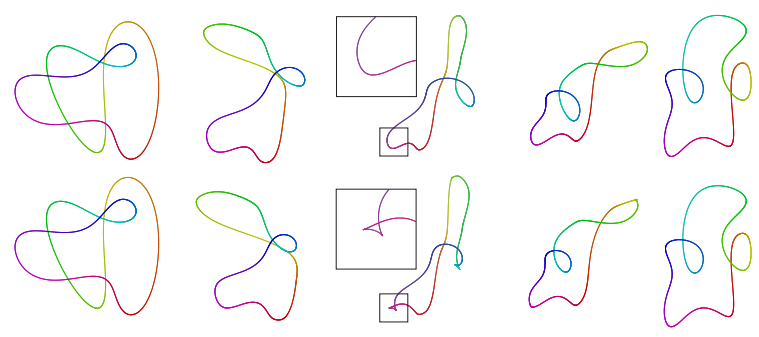

Figure 1: Our morph (top) is guaranteed to be a regular homotopy, while standard methods such as the angle-length method may display cusps as shown in the bottom row.

regular curves, called a regular homotopy, is possible if, and only if, the source and target curves have the same turning number (to be defined). Thus the non-regularity of the morph displayed in Figure 2 is unavoidable since the source and target curves have different turning numbers.

The goal of this paper is to devise an algorithm producing a visually pleasing regular homotopy $\mathbf{c}^{t}$ between two closed regular input curves $\mathbf{c}^{0}, \mathbf{c}^{1}$ with the same turning number. The importance of this goal is illustrated in the comparison with the angle-length method presented in Figure 1. We establish this goal by formulating a (non-linear) projection operator $\mathcal{P}$ onto the space of closed regular curves. The projection operator is defined via a convex representation of the 


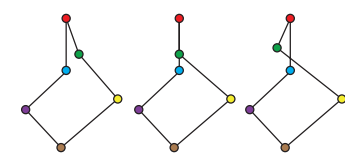

(a)

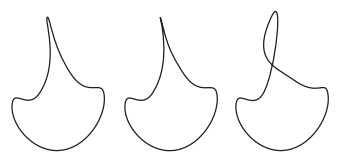

(b)
Figure 2: morphs between polygonal curves (a), and $C^{1}$ curves (b), with a non-regular intermediate curve.

space of regular closed curves with fixed external angles, together with a convex energy, defined over this space, measuring relative length deviation.

The projection operator is used to build the regular homotopy $\mathbf{c}^{t}$ as follows. First, we build intermediate 'ideal' curves $\overline{\mathbf{c}}^{t}$ defined so that the change in angles and length along the curve is monotone. These curves are of desired shape but are not closed in general. Second, we use the projection operator to 'close them' via solving a convex optimization problem, $\mathbf{c}^{t}=\mathcal{P}\left(\overline{\mathbf{c}}^{t}\right)$. The convexity of the projection operator, together with a feasibility result of the space of closed regular curves provides the proof of correctness of the algorithm.

The process of constructing ideal curves and then 'closing' them appear in several algorithms, e.g., the morphing algorithms in [SGWM93, SE02, SSHS14] and the fairing algorithm in [CPS13], however it is usually done by distributing the closing gap along all vertices without guaranteeing the regularity of the resulting closed curve. It is our belief that these algorithms can benefit from replacing the standard method of closing curves, with the method proposed here which guarantees regularity.

An important property of our algorithm is that when the source and target curves are convex, all intermediate curves will remain convex. The morph is also locally simple in a sense we define in section 6 , and it behaves well with respect to applying scaling, rigid motions, re-parameterizations and time reversal to the source and target curves.

We present several applications of our method; We extend our method to morph simple networks of curves; This allows the user to insert corresponding diagonals in source and target curves, thus better controlling the volume change during the morph. We show our algorithm can be modified to naturally morph between curves with different turning numbers with minimal pinching. We show that our method can be easily generalized to deal with convex combinations of more than two shapes. Finally, we give a sufficient condition for regular homotopic morphing of $b$-spline curves via morphing their control polygon using our algorithm. If the condition does not apply, it is possible to preform subdivisions of the source and target control polygons until the condition is fulfilled.

\section{Previous Work}

Polygonal curves. The most direct approach to morphing between polygonal curves is to linearly interpolate the ver- tices of the polygons. Early work in the field [SG92] showed that for certain correspondences between shapes this can cause severe problems, but suggested to choose a correspondence so that the linear morph will perform as little work as possible, in the sense of elasticity theory.

An improved strategy proposed by Sederberg and colleagues was the well known angle-length method [SGWM93], in which linear interpolation is conducted on the external angles and lengths of the polygonal curves, instead of on the vertices. As this does not result in a closed curve, the interpolated lengths are replaced with the closest lengths which do close the curve. Our general approach here is similar, but we preform the closing of the curve in such a way that regular homotopy is guaranteed, while Sederberg's algorithm sometimes fails to achieve this property.

Smooth curves. Piecewise $C^{1}$ curves are usually represented in some spline base, such as Bezier, b-splines or NURBS. If the correspondence problem is solved, so that the source and target curve are defined according to the same basis, and a correspondence is given between their respective control polygon, then morphing of the freeform curves can be conducted via morphing between the control polygons, using any one of the methods mentioned above. This strategy can be found in [SG95]. Since the control polygon is an 'exaggerated approximation' of the curve, this method may be less accurate than methods which exploit the properties of the curve itself.

Several methods (e.g., [MP06, KSMJ04] ) endow the space of curves with a riemannian structure, so that a morph between two curves can be found by computing the geodesic between them.

[SE02] morph between curves by morphing their curvature functions. The intermediate curves are closed heuristically by evenly distributing the 'error' between the vertices. [SSHS14] also linearly interpolate the curvature, and close the curve by choosing the closest curvature function which defines a closed curve. The discretization of this optimization problem results in a non-convex problem. Our method differs from the methods above in the closing technique which guarantees regular homotopy. Additionally, it isn't restricted to source and target curves which are parameterized by arc-length as these methods are.

Planar shapes. Morphing is often applied to compatible tessellated domains. In [SR95] the domains are compatibly partitioned into 'star-shaped polygons', which can be interpolated without self-intersections. [ACOL00] use consistent triangulations of shapes [ASS93] and suggest a pertriangle As-Rigid-As-Possible solution that is then stitched together to produce the final morph. More recent papers use different elasticity-inspired energies for defining the morph [HLZ04, BBA08]. Some methods define morphs based on metric interpolation [KMP07,WDAH10]. [CWKBC13] sug- 
gested bounded distortion morphing of domains based on conformal realization of intermediate domains.

Although these methods perform well in practice and possess certain guarantees, they still require a compatible tessellation of the domains and there is no proof that a compatible solution can be found once edge lengths (or any other defining quantity) is set, since the compatibility equations for triangulations are non-convex in general. As shown in this paper, for curves the situation is simpler and convex characterization exists.

Intersection free morphing. Several morphing algorithms assume that source and target curves are simple (nonintersecting), and construct morphs which are guaranteed to preserve simplicity. For example, [GS01] achieve this by equivalently triangulating the source and target shapes, and extending the triangulation to a common convex domain. [IOD09] preform intersection free morphing by combining a non-convex energy which prohibits intersection with a 'user supplied' energy which determines the properties of the morph. In contrast to intersection free morphing, a regular homotopy prohibits local intersections, but allows global intersections which may occur naturally in planar images (e.g., Figures 7, 13).

Regular homotopic fairing. [CPS13] propose an algorithm for regular homotopic fairing. In contrast to our method, the approach they suggest assumes fixed lengths, and closes intermediate curves by allowing modification of the angles.

Regular homotopy. The Whitney-Graustein Theorem [Whi37] says that there is a regular homotopy between two $C^{1}$ regular closed curves if and only if they have the same turning number. This Theorem was generalized to a much larger class of curves, including those we discuss here, by [MH47]. [MY91] construct a regular homotopy between polygonal curves in which each intermediate curve is a polygon. Their proof is rather complex and our presentation here provides an alternative proof which is arguably simpler. A proof along these lines also appears in [Pin10].

\section{Preliminaries and problem statement}

Closed regular curves. We will discuss curves $\mathbf{c}:[0,1] \rightarrow$ $\mathbb{R}^{2}$ fulfilling the following conditions:

1. Closed. We consider closed curves, that is $\mathbf{c}(0)=\mathbf{c}(1)$.

2. Continuous, Piecewise $C^{1}$. $\mathbf{c}$ is continuous, and its derivative $\dot{\mathbf{c}} \equiv \frac{d \mathbf{c}}{d s}$ exists and is continuous in all but a finite set of singular points $\mathfrak{s}_{1}, \ldots, \mathfrak{s}_{n} \in[0,1)$. At these points the right $\dot{\mathbf{c}}_{+}$and left $\dot{\mathbf{c}}_{-}$limits of $\dot{\mathbf{c}}$ exist. We define $\dot{\mathbf{c}}_{+}(1)=\dot{\mathbf{c}}_{+}(0)$, and $\dot{\mathbf{c}}_{-}(0)=\dot{\mathbf{c}}_{-}(1)$.

3. Regular. The curve's derivative $\dot{\mathbf{c}}$ is non-zero except possibly at the singular points. At the singular points $\mathfrak{s}_{j}$, the left and right limits $T_{-}$and $T_{+}$of the tangent field $T=\frac{\dot{\mathbf{c}}}{|\dot{\mathbf{c}}|}$ exist, and satisfy $T_{-}\left(\mathfrak{s}_{j}\right) \neq-T_{+}\left(\mathfrak{s}_{j}\right)$.
We note that when there are no singular points our definition coincides with the standard definition of regular $C^{1}$ curves, and that when $\mathbf{c}$ is piecewise linear our definition coincides with the standard definition of regular polygonal curves.

Figure 2 shows non-regular closed curves in (a)-middle and (b)-middle.

Turning number and regular homotopy. The external angle $\theta_{j}$ at a singular point $\mathfrak{s}_{j}$ is the unique angle in $(-\pi, \pi)$ satisfying $e^{i \theta_{j}} T_{-}\left(\mathfrak{s}_{j}\right)=T_{+}\left(\mathfrak{s}_{j}\right)$, as shown in the inset. Since $\mathbf{c}$ is regular $\theta_{j}$ is well-defined. It is convenient to represent the curve's speed in polar form with complex numbers $\dot{\mathbf{c}}(s)=r(s) e^{i \varphi(s)}$, where $r, \varphi:[0,1] \rightarrow \mathbb{R}$, are piecewise $C^{1}$ functions (not continuous in general), and $r>0 . \varphi$ is uniquely defined

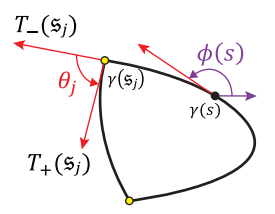
up to addition of a constant function whose value is an integer multiply of $2 \pi$ by the requirement that at singularities $\varphi$ will satisfy

$$
\varphi_{+}\left(\mathfrak{s}_{j}\right)=\varphi_{-}\left(\mathfrak{s}_{j}\right)+\theta_{j}
$$

If $\mathbf{c}$ is a closed curve, then $\dot{\mathbf{c}}_{+}(0)=\dot{\mathbf{c}}_{+}(1)$ and therefore there is an integer $\tau(\mathbf{c})$ called the turning number of $\mathbf{c}$, such that

$$
2 \pi \tau(\mathbf{c})=\varphi_{+}(1)-\varphi_{+}(0)
$$

Below we show several examples of closed regular curves and their turning number.
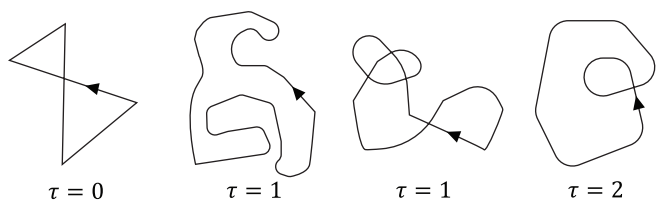

We note that it is known that simple closed curves $\mathbf{c}$ with positive orientation have $\tau(\mathbf{c})=1$. As a result the turning number of many examples of interest is one.

Given two closed regular curves $\mathbf{c}^{0}(s), \mathbf{c}^{1}(s):[0,1] \rightarrow \mathbb{R}^{2}$, a morph (or homotopy) $\mathbf{c}^{t}(s)=\mathbf{c}(t, s):[0,1] \times[0,1] \rightarrow \mathbb{R}^{2}$ is a continuous function in $t, s$ such that for $t=0, t=1$ it coincides with $\mathbf{c}^{0}, \mathbf{c}^{1}$, respectively. A morph where for all $t, \mathbf{c}^{t}$ is a closed regular curve is called a regular homotopy. The Whitney-Graustein Theorem [Whi37] asserts that there exists a regular homotopy between a pair of closed regular curves $\mathbf{c}^{0}, \mathbf{c}^{1}$ if and only if they have the same turning number, $\tau\left(\mathbf{c}^{0}\right)=\tau\left(\mathbf{c}^{1}\right)$. For example, there exists a regular homotopy between the second and third curves in the figure above, and there is no regular homotopy between all other pairs of curves in that figure.

Problem statement. The goal of this paper is to devise an algorithm that, given two closed regular curves $\mathbf{c}^{0}, \mathbf{c}^{1}$ : 
$[0,1] \rightarrow \mathbb{R}^{2}$ with the same turning number, constructs a visually pleasing regular homotopy $\mathbf{c}^{t}:[0,1] \rightarrow \mathbb{R}^{2}$ of the curves.

\section{Method}

This section develops the algorithm to solve the regular homotopy problem. The key idea is to construct an "ideal curve" $\overline{\mathbf{c}}^{t}$ for time $t$ and to project it onto the space of closed regular curves, $\mathbf{c}^{t}=\mathcal{P}\left(\overline{\mathbf{c}}^{t}\right)$, using a convex characterization of that space.

Ideal Intermediate Curves. A natural approach to construct a regular homotopy between $\mathbf{c}^{0}, \mathbf{c}^{1}$ would be to linearly interpolate angles and lengths of the two curves. This can be done using the polar representation of the curves' derivatives $\varphi^{0}, r^{0}$ and $\varphi^{1}, r^{1}$,

$$
\bar{\varphi}^{t}=(1-t) \varphi^{0}+t \varphi^{1} \quad \bar{r}^{t}=(1-t) r^{0}+t r^{1}
$$

If the length of $\mathbf{c}^{0}, \mathbf{c}^{1}$ is not restricted to unity, the $\bar{r}^{t}(s)$ will be biased towards the curve whose total length is larger. We therefore normalize all curve lengths to be one, so that $r^{t}$ is in fact the speed of the curve $\mathbf{c}^{t}$ relative to its total length.

The ideal curve at a fixed time $t$ can be reconstructed by integrating the derivative of the curve, that is let $\dot{\overline{\mathbf{c}}}^{t}(s)=$ $\bar{r}^{t}(s) e^{i \bar{\varphi}^{t}(s)}$, and

$$
\overline{\mathbf{c}}^{t}(s)=\overline{\mathbf{c}}^{t}(0)+\int_{0}^{s} \dot{\overline{\mathbf{c}}}^{t}
$$

where $\overline{\mathbf{c}}^{t}(0) \in \mathbb{R}^{2}$ is a translational degree of freedom. The curve $\overline{\mathbf{c}}^{t}$ defined in eq. (4.1) is regular by construction but is not, in general, closed. We will consider $\left(\bar{r}^{t}, \bar{\varphi}^{t}\right)$ the 'ideal' solution, and choose a solution as close as possible to it, in a sense to be defined, from the space of closed regular curves.

The motivation for our definition of ideal curves, is that the change in length and angle along sub-arcs, defined for fixed $s_{1}, s_{2} \in[0,1]$ by

$$
\begin{gathered}
L\left(\overline{\mathbf{c}}^{t}, s_{1}, s_{2}\right)=\int_{s_{1}}^{s_{2}}\left|\dot{\overline{\mathbf{c}}}^{t}(s)\right| d s=\int_{s_{1}}^{s_{2}} \bar{r}^{t}(s) d s \\
\operatorname{angle}\left(\overline{\mathbf{c}}^{t}, s_{1}, s_{2}\right)=\int_{s_{1}}^{s_{2}} \dot{\bar{\varphi}}^{t}=\bar{\varphi}^{t}\left(s_{2}\right)-\bar{\varphi}^{t}\left(s_{1}\right)
\end{gathered}
$$

change linearly in $t$. This generalizes the ideal curves chosen by the angle-length method [SGWM93] for polygons.

Closed regular curves. Curves $\mathbf{c}^{t}$ constructed from piecewise $C^{1}$ functions $\left(r^{t}, \varphi^{t}\right)$ are regular, if for all $t$,

$$
\begin{aligned}
& \mathbf{c}^{t}(1)-\mathbf{c}^{t}(0)=\int_{0}^{1} \dot{\mathbf{c}}^{t}=\int_{0}^{1} r^{t}(s) e^{i \varphi^{t}(s)} d s=0 \\
& r^{t}>0 \\
& \left|\varphi_{+}^{t}\left(\mathfrak{s}_{j}\right)-\varphi_{-}^{t}\left(\mathfrak{s}_{j}\right)\right|<\pi, \quad \forall j
\end{aligned}
$$

In general, these conditions are non-convex in $\left(\varphi^{t}, r^{t}\right)$ and hence difficult to work with. Inspired by [SGWM93] we propose to fix $\varphi^{t}=\bar{\varphi}^{t}$ and leave only $r^{t}$ free. Since $\bar{\varphi}^{t}$ is a convex combination of $\varphi^{0}$ and $\varphi^{1}, \varphi^{t}$ satisfies (4.2c). This renders eqs. (4.2) convex (in $r^{t}$ ) but raises the question if this is not too strong of a constraint, that is, is (4.2) still feasible? Namely, is it always possible to find a positive length function $r^{t}>0$ such that $\left(r^{t}, \varphi^{t}\right)$ define a closed regular curve? The answer is affirmative as formulated in the next Theorem.

Theorem 4.1. If $\tau\left(\mathbf{c}^{0}\right)=\tau\left(\mathbf{c}^{1}\right) \neq 0$, then for all $t \in[0,1]$ there exists piecewise $C^{1}$ function $r^{t}$ satisfying (4.2a),(4.2b).

We discuss the case of zero turning number when we prove the Theorem in Appendix A. Let us denote

$$
\Gamma^{t}=\left\{r^{t} \mid r^{t} \text { satisfies (4.2a), (4.2b) }\right\}
$$

the non-empty set of feasible solutions $r^{t}$ of (4.2), given $\varphi^{t}=$ $\bar{\varphi}^{t}$.

Energy. We now want to search for the member of the convex non-empty set $\Gamma^{t}$ that is closest to $\bar{r}^{t}$ with respect to some energy $E\left(r^{t}, \bar{r}^{t}\right)$. The energy we propose is

$$
E\left(r^{t}, \bar{r}^{t}\right)=\int_{0}^{1}\left(\frac{r^{t}(s)-\bar{r}^{t}(s)}{\sqrt{\bar{r}^{t}(s) r^{t}(s)}}\right)^{2} d \ell(s)
$$

This is the relative length distortion with respect to the ideal curve, integrated according to the length element $d \ell(s)=\frac{1}{2}\left(r^{t}(s)+\bar{r}^{t}(s)\right) d s$, which is the average of the length element of the ideal curve and the length element of the closed curve to be constructed.

Another interpretation of the energy can be obtained, using the fact that the curvature $\kappa$ of a regular curve $\mathbf{c}$ is a simple function of the polar coordinates of its speed, i.e., $\kappa=\frac{\dot{\varphi}}{r}$. Thus, denoting the curvature of the curves defined by $\left(\bar{\varphi}^{t}, \bar{r}^{t}\right)$ and $\left(\bar{\varphi}^{t}, r^{t}\right)$ by $\bar{\kappa}^{t}$ and $\kappa^{t}$ respectively, we have

$$
E\left(r^{t}, \bar{r}^{t}\right)=\int_{0}^{1}\left(\frac{\kappa^{t}(s)-\bar{\kappa}^{t}(s)}{\sqrt{\bar{\kappa}^{t}(s) \kappa^{t}(s)}}\right)^{2} d \ell(s)
$$

We note that $E\left(r^{t}, \bar{r}^{t}\right)$ is of the form

$$
E\left(r^{t}, \bar{r}^{t}\right)=\int_{0}^{1} f\left(r^{t}(s)\right) d s
$$

where $f$ can be rewritten as

$$
f(r)=\frac{1}{2}\left(\frac{1}{\bar{r}^{t}} r^{2}-r-\bar{r}^{t}+\left(\bar{r}^{t}\right)^{2} \frac{1}{r}\right)
$$

Thus $f(r)$ is infinite when $r=0$, and $\frac{d^{2} f}{d r^{2}}>0$ for $r>0$. Therefore the energy $E\left(r^{t}, \bar{r}^{t}\right)$ is strictly convex over $\Gamma^{t}$ and explodes as $r^{t}$ approaches zero. Intuitively, the relative distance functional $E$ turns out to be convex when restricted to the space of closed regular curves $\Gamma^{t}$ and naturally discourage the speed $r^{t}$ of the curves $\mathbf{c}^{t}$ from approaching zero. 
Convex projection operator. Finally, we state the full formulation of our optimization problem

$$
\begin{aligned}
& \min _{r^{t}} E\left(r^{t}, \bar{r}^{t}\right) \\
& \text { s.t. } \\
& r^{t}>0 \\
& \int_{0}^{1} r^{t}(s) e^{i \varphi^{t}(s)} d s=0 \\
& \int_{0}^{1} r^{t}(s) d s=1
\end{aligned}
$$

where (4.3d) restricts the curve to unit length to maintain consistency with our interpretation of $r^{t}$ as the speed of the curve relative to its total length. The unique solution $r^{t}$ of (4.3) is now used to define $\dot{\mathbf{c}}^{t}=r^{t} e^{i \bar{\varphi}^{t}}$ which is then integrated (see Eq. (4.1)) to obtain $\mathbf{c}^{t}=\mathcal{P}\left(\overline{\mathbf{c}}^{t}\right)$.

In practice we approximate the solution of (4.3) by choosing a dense partition $0=\mathfrak{s}_{0}<\mathfrak{s}_{1}<\ldots<\mathfrak{s}_{n}=1$ (which includes the original singular points of the curves) and then approximating $\mathbf{c}^{m}, m=0,1$, by the piecewise linear curve $\mathbf{c}_{n}^{m}$ whose singular points are $\mathfrak{s}_{i}$, and whose value at $\mathfrak{s}_{i}$ is $\mathbf{c}^{m}\left(\mathfrak{s}_{i}\right)$. For sufficiently dense partitions, $\mathbf{c}_{n}^{m}$ will be regular curves with the same turning number as $\mathbf{c}^{m}$ (see supplementary material).

After approximating the source and target curves, we search for solutions $\mathbf{c}^{t}$ which are also piecewise linear with the same singular points. This reduces to the finite dimensional optimization problem of morphing polygons which we will now describe.

\section{The polygonal case}

In this section we focus on the polygonal curve case. We show that in this case the optimization problem (4.3) boils down to a Second-Order Cone Program (SOCP), and we provide all details of the morphing algorithm. We consider the source and target closed regular polygons

$$
\mathbf{p}^{m}=\left[\mathbf{p}_{1}^{m}, \mathbf{p}_{2}^{m}, \ldots, \mathbf{p}_{n}^{m}=\mathbf{p}_{1}^{m}\right], \quad m=0,1
$$

where $\mathbf{p}_{j}^{m} \in \mathbb{R}^{2}$. Our goal is to produce a visually pleasing regular homotopy of closed polygonal curves $\mathbf{p}^{t}=$ $\left[\mathbf{p}_{1}^{t}, \ldots, \mathbf{p}_{n}^{t}=\mathbf{p}_{1}^{t}\right]$.

The source and target polygons $\mathbf{p}^{m}, m=0,1$, can be interpreted as piecewise-linear regular closed curves by choosing any $n$ singular points in the unit interval $0=\mathfrak{s}_{1}<\mathfrak{s}_{2}<$ $\ldots<\mathfrak{s}_{n}=1$ (the interpolation constructed will be independent of this choice) and defining $\mathbf{c}^{m}, m=0,1$ to be the unique piecewise-linear curves such that $\mathbf{c}^{m}\left(\mathfrak{s}_{j}\right)=\mathbf{p}_{j}^{m}$, for all $j=1, \ldots, n$. We can then construct intermediate curves $\mathbf{c}^{t}$ using (4.3). We note that $\bar{r}^{t}$ and $\varphi^{t}=\bar{\varphi}^{t}$ are constant on the intervals $\Delta_{j}=\left[\mathfrak{s}_{j}, \mathfrak{s}_{j+1}\right]$, and strict convexity implies that the minimizer $r^{t}$ of (4.3) is constant on these intervals as well, so that $\mathbf{c}^{t}$ is in fact piecewise linear and defines a polygon $\mathbf{p}^{t}$ $\operatorname{via} \mathbf{p}_{j}^{t}=\mathbf{c}^{t}\left(\mathfrak{s}_{j}\right)$.
We define $L_{j}^{t}, \bar{L}_{j}^{t}, \varphi_{j}^{t} \in \mathbb{R}$ by the equations

$$
\left.r^{t}\right|_{\Delta_{j}}=\frac{L_{j}^{t}}{\left|\Delta_{j}\right|} ;\left.\bar{r}^{t}\right|_{\Delta_{j}}=\frac{\bar{L}_{j}^{t}}{\left|\Delta_{j}\right|} ;\left.\varphi^{t}\right|_{\Delta_{j}}=\varphi_{j}^{t}
$$

Plugging $r^{t}, \bar{r}^{t}, \varphi^{t}$ into (4.3) gives a finite dimensional optimization problem:

$$
\begin{array}{cl}
\min _{L^{t}} & \sum_{j}\left(\frac{L_{j}^{t}-\bar{L}_{j}^{t}}{\sqrt{\bar{L}_{j}^{t} L_{j}^{t}}}\right)^{2} \frac{\bar{L}_{j}^{t}+L_{j}^{t}}{2} \\
\text { s.t. } & L_{j}^{t} \geq 0 \\
& \sum_{j} L_{j}^{t} e^{i \bar{\varphi}_{j}^{t}}=0 \\
& \sum_{j} L_{j}^{t}=1
\end{array}
$$

The feasibility of this optimization problem is a discrete version of Theorem 4.1 stated here and proved at the end of this section,

Theorem 5.1. If $\tau\left(\mathbf{p}^{0}\right)=\tau\left(\mathbf{p}^{1}\right) \neq 0$, then for all $t \in[0,1]$ the polygonal regular homotopy optimization problem (5.1) is always strictly feasible. That is, has a strictly positive solution $L_{j}^{t}>0$.

The unique minimum $L^{t}=\left(L_{j}^{t}\right)_{j=1}^{n-1}$ of the strictly convex functional satisfying the constraints is strictly positive since (5.1a) is $+\infty$ if some $L_{j}^{t}=0$. Furthermore, as we prove in Appendix A,

Lemma 5.2. $L_{j}^{t}$ is a $C^{\infty}$ function of $t$.

We conclude that the morph $\mathbf{c}^{t}$ defined up to translation by $\left(L_{j}^{t}, \bar{\varphi}_{j}^{t}\right)$ is a regular homotopy. In fact, the vertex paths $\mathbf{c}^{t}\left(\mathfrak{s}_{j}\right)$ are smooth in $t$.

Recast as SOCP. We now show how (5.1) can be rephrased as a second order cone program (SOCP) for which several standard solvers are available. We regroup (5.1a) to obtain

$$
\frac{1}{2} \sum_{j} \frac{1}{\bar{L}_{j}^{t}}\left(L_{j}^{t}\right)^{2}-L_{j}^{t}-\bar{L}_{j}^{t}+\left(\bar{L}_{j}^{t}\right)^{2} \frac{1}{L_{j}^{t}}
$$

We replace the expressions $1 / L_{j}^{t}$ with the slack variables $\eta_{j}^{t}$ and add the constraint $1 / L_{j}^{t} \leq \eta_{j}^{t}$ to obtain an equivalent formulation for (5.1):

$$
\begin{aligned}
& \min _{L^{t}, \eta^{t}} \frac{1}{2} \sum_{j} \frac{1}{\bar{L}_{j}^{t}}\left(L_{j}^{t}\right)^{2}-L_{j}^{t}-\bar{L}_{j}^{t}+\left(\bar{L}_{j}^{t}\right)^{2} \eta_{j}^{t} \\
& \text { s.t. } \\
& \quad \eta_{j}^{t} L_{j}^{t} \geq 1 \\
& \quad L_{j}^{t} \text { satisfy the constraints }(5.1 \mathrm{~b})-(5.1 \mathrm{~d})
\end{aligned}
$$

This formulation is equivalent since if $\left(L^{t}, \eta^{t}\right)$ is the minimizer of (5.2), then (5.2b) will necessarily be active. (5.2b) 
is a rotated cone condition, which can be formulated as the second order cone condition

$$
\eta_{j}^{t}+L_{j}^{t} \geq\left\|\left(2, L_{j}^{t}-\eta_{j}^{t}\right)\right\|_{2}
$$

Curve reconstruction. Once the solution $L^{t}$ to (5.1) is found, the polygonal curve $\mathbf{p}^{t}$ can be reconstructed by integrating the derivative $\dot{\mathbf{c}}^{t}$ as defined in Eq. (4.1). This can be done by solving the linear system of equations

$$
\mathbf{p}_{j+1}^{t}-\mathbf{p}_{j}^{t}=L_{j}^{t} e^{i \bar{\varphi}_{j}^{t}}
$$

that is uniquely solvable up-to a translational degree of freedom. We fix this degree of freedom by requiring that the center of mass of each intermediate curve

$$
C^{t}=\sum_{j=1}^{n} \frac{L_{j}^{t}+L_{j-1}^{t}}{2} \mathbf{p}_{j}^{t}
$$

(where we define $L_{n}^{t}=L_{1}^{t}$ ) will be the linear interpolation of $C^{0}$ and $C^{1}$.

Scaling. Since we rescale the source and target curves to have unit length (see section 4), the morph $\mathbf{c}^{t}$ needs to be scaled back to the original dimension of the problem. We choose a continuous scaling $S:[0,1] \rightarrow \mathbb{R}_{+}$for $\mathbf{c}^{t}$ so that $S(m) \mathbf{c}^{m}, m=0,1$ is the original curve $\tilde{\mathbf{c}}^{m}$ supplied by the user before scaling. We use the linear interpolation of the diameter of the shape, that is

$$
S(m)=\operatorname{diam}\left(\tilde{\mathbf{c}}^{m}\right), \quad S(t)=(1-t) S(0)+t S(1)
$$

Choosing $\varphi_{1}^{m}$. For regular curves, the choice of $\varphi_{1}^{m}, m=$ 0,1 defines $\varphi_{1}^{t}$, and thus all of $\varphi_{j}^{t}$, uniquely. To choose this initial condition we begin with $\tilde{\varphi}_{1}^{0}, \tilde{\varphi}_{1}^{1} \in(-\pi, \pi)$, and then search for the $k \in \mathbb{Z}$ minimizing the work in angle space, measured with respect to the average discrete length element $\bar{L}^{\frac{1}{2}}=\frac{1}{2}\left(L^{0}+L^{1}\right)$ :

$$
k^{*}=\min _{k \in \mathbb{Z}} \sum_{j=1}^{n-1}\left[\left(\tilde{\varphi}_{j}^{1}+2 \pi k\right)-\tilde{\varphi}_{j}^{0}\right]^{2} \bar{L}_{j}^{\frac{1}{2}}
$$

We then choose

$$
\varphi_{1}^{0}=\tilde{\varphi}_{1}^{0}, \varphi_{1}^{1}=\tilde{\varphi}_{1}^{1}+2 \pi k^{*}
$$

We summarize the polygonal morphing algorithm in Algorithm 1.

Feasibility of the optimization problem. We show that the optimization problem (5.1) is strictly feasible as Theorem 5.1 above states. The proof also contains the main idea for the proof of the continuous case which is presented in full detail in Appendix A.

The main tool for the feasibility proof is the following Lemma,

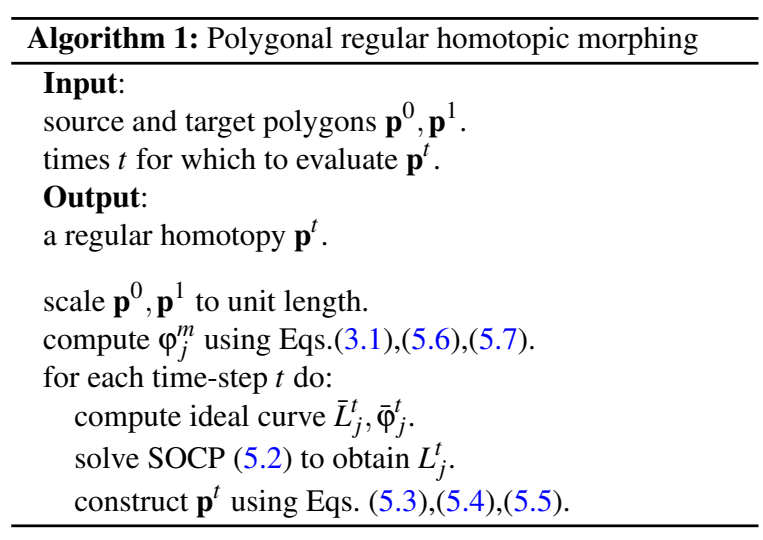

Lemma 5.3. Let $\varphi_{1}, \ldots, \varphi_{n-1} \in \mathbb{R}$ be a sequence of real numbers with maximal and minimal elements $\varphi_{\max }, \varphi_{\min }$, satisfying

$$
\left|\varphi_{j+1}-\varphi_{j}\right|<\pi, \varphi_{\max }-\varphi_{\min }>\pi
$$

Then

1. the conical hull Coni $\left\{e^{i \varphi_{j}}: j=1, . ., n-1\right\}$ spans all of the plane.

(For a set $\left.A \subseteq \mathbb{R}^{n}, \operatorname{Coni}(A)=\left\{\sum \eta_{i} a_{i} \mid a_{i} \in A, \eta_{i} \geq 0\right\}.\right)$

2. There is a strictly positive solution $L_{j}>0$ to the equation $\sum L_{j} e^{i \varphi_{j}}=0$.

The first part of the Lemma is proved in Appendix A, however the idea of the proof is that the conditions on the numbers $\left\{\varphi_{j}\right\}$ imply that the points on the unit circle $\left\{e^{i \varphi_{j}}\right\}$ do not leave an empty half-circle and therefore their conichull covers the plane. See figure 3 for an illustration of this idea.

The second part is an immediate conclusion from the first part. Using the first part, we can choose $\tilde{L}_{j} \geq 0$ such that $\sum \tilde{L}_{j} e^{i \varphi_{j}}=-\sum e^{i \varphi_{j}}$. Rearranging this expression we see that $L_{j}=\tilde{L}_{j}+1$ is a positive solution of $\sum L_{j} e^{i \varphi_{j}}=0$.
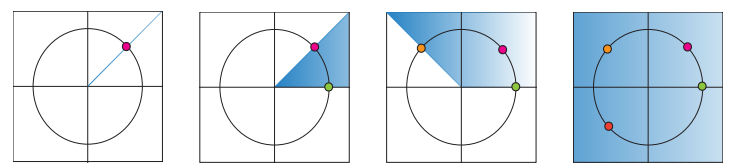

Figure 3: Proof of feasibility. The conical hull of the unit vectors marked by colored disks is the region colored in blue. In each figure a new vector $e^{i \varphi_{j}}$ is added $(\varphi=$ $\left.\left(\frac{\pi}{4}, 0, \frac{3}{4} \pi, \frac{5}{4} \pi\right)\right)$. The conical hull in the last figure is $\mathbb{R}^{2}$ since $\varphi_{4}-\varphi_{2}>\pi$.

To conclude the feasibility proof, it is sufficient to show that $\varphi_{j}^{t}, j=1, \ldots, n-1$ satisfy the conditions of the Lemma. If so, by the second part of the Lemma there are positive lengths $L_{j}^{t}$ which satisfy the curve closing equation (5.1c), 
and this solution can be normalized so that it also satisfies the unit length condition (5.1d).

To see that $\varphi_{j}^{t}$ satisfy the conditions of the Lemma, consider the angle function $\varphi^{m}$ of the piecewise-linear curve $\mathbf{c}^{m}$ defined by $\mathbf{p}^{m}, m=0,1$. Denote $\tau \equiv \tau\left(\mathbf{c}^{0}\right)=\tau\left(\mathbf{c}^{1}\right)$. Using (3.1) and the definition of the turning number in (3.2), we obtain

$$
\begin{aligned}
2 \pi \tau & =\varphi_{+}^{m}(1)-\varphi_{+}^{m}(0) \\
& =\left(\varphi_{+}^{m}(1)-\varphi_{-}^{m}(1)\right)+\left(\varphi_{-}^{m}(1)-\varphi_{+}^{m}(0)\right) \\
& =\theta_{n}+\varphi_{n-1}^{m}-\varphi_{1}^{m}
\end{aligned}
$$

Since $\left|\theta_{n}\right|<\pi$, it follows that for $m=0,1, \varphi_{n-1}^{m}-\varphi_{1}^{m}$ is in the convex set $(2 \pi \tau-\pi, 2 \pi \tau+\pi)$, and therefore so are $\varphi_{n-1}^{t}-\varphi_{1}^{t}, t \in[0,1]$. In particular $\left|\varphi_{n-1}^{t}-\varphi_{1}^{t}\right|>\pi$ and so $\varphi_{j}^{t}, \quad j=1, \ldots, n-1$ satisfy the conditions of the Lemma.

\section{Properties}

We now present several properties of the regular homotopy $\mathbf{c}^{t}=\mathcal{P}\left(\overline{\mathbf{c}}^{t}\right)$.

Smooth vertex path. As discussed earlier, the trajectory of the vertices $\mathbf{p}_{j}^{t}$ is a $C^{\infty}$ function of $t$ (Lemma 5.2).

Scaling and rigid motion invariance. If source and target curves $\mathbf{c}^{m}, m=0,1$ undergo a scaling and/or rigid motion then $\mathbf{c}^{t}$ will undergo a transformation of the same nature. This property is due to the fact that the optimization problems (4.3),(5.1) are formulated only in terms of angles and lengths and the particular scaling procedure employed.

Invariance to change of parameter. If $\mathbf{c}_{*}^{m}$ are obtained from $\mathbf{c}^{m}$ by an identical change of parameter, i.e.,

$$
\mathbf{c}_{*}^{m}=\mathbf{c}^{m} \circ p, m=0,1
$$

where $p:[0,1] \rightarrow[0,1]$ is strictly monotonically increasing and piecewise $C^{1}$, then the morph satisfies $\mathbf{c}_{*}^{t}=\mathbf{c}^{t} \circ p$. This is a natural property since joint reparameterization doesn't change the correspondence between points on the curves, and so the intermediate curves should also remain the same, up to the reparameterization. This property can be explained by noting that the change of variables $s=p(\sigma)$ in the integrals in (4.3) leads to an equivalent problem and therefore $r^{t}(s)$ is the solution of (4.3) iff $r_{*}^{t}(\sigma)=r(p(\sigma)) p^{\prime}(\sigma)$ is the solution of (4.3) with $\mathbf{c}_{*}^{m}$ used as input curves.

In the polygonal case this implies that subdividing an edge by inserting a new vertex does not change the polygons created throughout the morph. This property is sometimes referred to as identity preservation.

Time reversal. If $\mathbf{c}^{0}$ and $\mathbf{c}^{1}$ switch roles, that is $\mathbf{c}_{*}^{0}=\mathbf{c}^{1}$ and $\mathbf{c}_{*}^{1}=\mathbf{c}^{0}$, then $\mathbf{c}_{*}^{t}=\mathbf{c}^{1-t}$.
Convexity preservation. If $\mathbf{c}^{0}, \mathbf{c}^{1}$ are simple convex curves (simple closed regular curves bounding convex regions), the intermediate curves $\mathbf{c}^{t}$ are guaranteed to be convex as well. This follows from

Lemma 6.1. A closed regular curve $\mathbf{c}$ with $\tau(\mathbf{c})=1$ is convex, iff $\varphi$ is a non decreasing function.

Therefore, if $\mathbf{c}^{0}, \mathbf{c}^{1}$ are convex, $\varphi^{0}, \varphi^{1}$ will be nondecreasing, and as a result $\varphi^{t}=\bar{\varphi}^{t}$ will also be nondecreasing, and so $\mathbf{c}^{t}$ will be convex. The proof of the Lemma appears in the supplementary material.

Locally non-intersecting. If $\mathbf{c}^{0}, \mathbf{c}^{1}$ are simple and nonconvex it does not imply in general that $\mathbf{c}^{t}$ is simple as-well. However, we can guarantee that monotone sub-arcs of the curve stay simple. We say that a curve $\mathbf{c}$ is monotone along $\left[s_{1}, s_{2}\right]$, if for all non-singular $s, s^{\prime} \in\left[s_{1}, s_{2}\right]$,

$$
\varphi(s)-\varphi\left(s^{\prime}\right) \leq \pi
$$

This is equivalent to saying that $e^{i \varphi}$ are all in the half circle defined by some $e^{i \alpha}$, which implies that $s \mapsto\left\langle e^{i \alpha}, \mathbf{c}(s)\right\rangle$ is non-decreasing along $\left[s_{1}, s_{2}\right]$, or in other words, that $\mathbf{c}$ is monotone along $\left[s_{1}, s_{2}\right]$ in the direction $e^{i \alpha}$ and cannot self-intersect. The cyan curve displayed at the right is monotone in the direction $e^{0}$, and the red curve is a monotone in the direction $e^{i \frac{\pi}{2}}$. If $\mathbf{c}^{0}, \mathbf{c}^{1}$ are both monotone along $\left[s_{1}, s_{2}\right]$, then since (6.1) is a convex condition, so are the intermediate curves $\mathbf{c}^{t}$. This implies that $\left.\mathbf{c}^{t}\right|_{\left[s_{1}, s_{2}\right]}$ is simple for all $t \in[0,1]$. Thus our morphing method is locally non-intersecting in the sense that intersections cannot occur in sub-intervals in which the tangent fields of the source and target curves does not change substantially.

\section{Results}

In this section we provide evaluation of our curve morphing algorithm, and compare it to relevant previous methods.

Curves from standard datasets. We verified that our method produces high quality morphs by running our algorithm on examples from standard datasets (Figures 4 and 5). We found the correspondence between the shapes by initially using the algorithm of [LJ07], and then slightly modifying the correspondence of a small number of points manually to increase the accuracy of the correspondence, when necessary. To illustrate the correspondence, the curves are colored so that corresponding regions in different curves are colored identically. Source and target are left and right curves, respectively.

Comparison with curve-based methods. We compared our method with the angle-length method [SGWM93] and with the curvature morph method [SSHS14].

A disadvantage of the curvature method is the requirement that source and target curves are parameterized by arclength, which is not necessarily the natural correspondence 


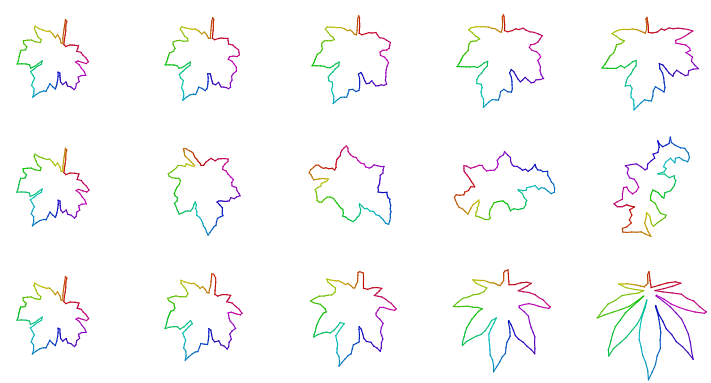

Figure 4: Morphs of leaves from [TvW02]
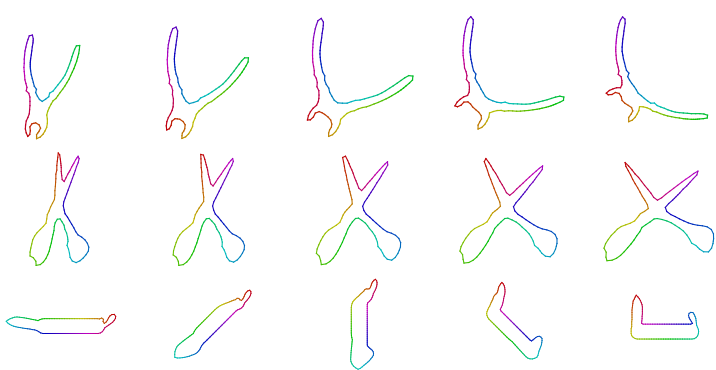

Figure 5: Morphs of tools from [BBBK08]. The correspondence for the pocket knife is given so that the different blades deform from one to the other.

to choose, as can be seen in Figure 6: The source curve is a cubic b-spline generated by a square with three added vertices evenly distributed along each edge, and the target is obtained from the source by scaling the $x$-axis of the control polygon. This defines a natural correspondence between the control polygons and hence between the splines. However, the curvature algorithm (in (b)) reparameterizes both curves in arc-length parameterization, and as a result returns intermediate curves with more than four 'vertices'. The curvature method also fails to preserve the shape of the kite in Figure 7.

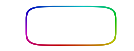

(a)

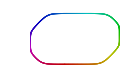

(b)

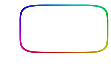

(c)
Figure 6: Comparison of (a) angle-length (b) curvature and (c) our algorithm. The requirement that source and target curves are parameterized by arc-length creates new vertices in the intermediate curves, as opposed to the natural morph generated by angle-length interpolation methods.

Figures 7 also shows that the angle-length method can produce curves with cusps which our algorithm is guaranteed to avoid. In Figure 8 we morph two convex quads and compare with the angle-length method for polygons. The angle-length method changes the turning number throughout the morph, resulting in an irregular morph, while our

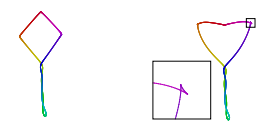

(a)

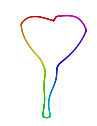

(b)

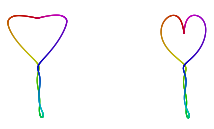

(c)
Figure 7: Comparison of (a) angle-length (b) curvature and (c) our algorithm. Note the cusp produced by the anglelength method.

morph is guaranteed to return a convex curve throughout the morph. The reason for the irregular behavior of the anglelength method in these examples seems to be the weighting chosen in [SGWM93], which in this case effectively forces one of the quad edges to remain constant throughout the morph. We have found that also if the weighting is changed to various other natural options, there are example in which the turning number isn't preserved. In Figure 9 we show such an example for a choice of uniform weights.

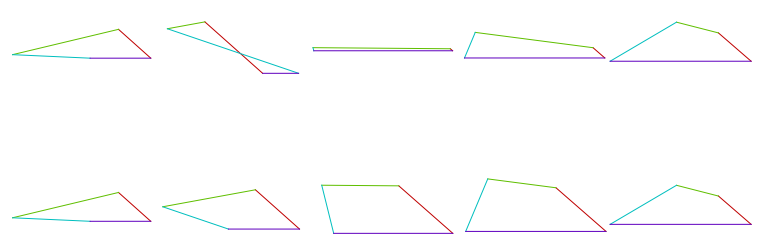

Figure 8: Comparison of angle-length (top) with our method. The turning number of the angle-length method changes during the morph, causing the intersection in the second figure from the left, while our method is guaranteed to preserve convexity.

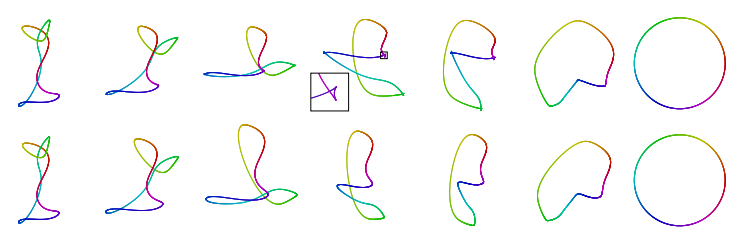

Figure 9: The angle-length method (top) exhibits cusps while our algorithm produces a regular homotopy.

Comparison with tessellation-based methods. In Figure 10 we compare our method with the bounded-distortion triangular mesh morphing method of [CWKBC13]. Both methods return agreeable and very similar results. It seems that morphing of domains can be carried out, in many cases, without necessarily utilizing any information on the interior of the shapes. We believe, based on our experiments, that this is the case in most morphing problems. In section 8 we propose a generalization of our method which can handle the infrequent cases where some interior information is needed.

Timing. We implemented our algorithm on Matlab, using 


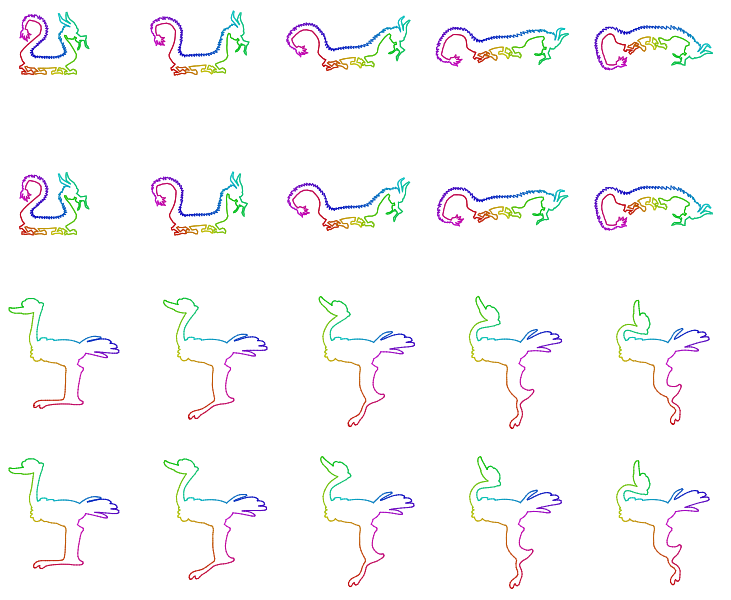

Figure 10: Comparison of morphing using triangular meshes (top row in both examples), with our method. Both methods give similar natural results, suggesting that the morphing problem can be solved without information on the interior of the shape.

Yalmip [Lof04] for the formulation of the SOCP optimization problem and Mosek [AA00] to solve the SOCP optimization. Running our algorithm on a $C^{1}$ curve sampled at 2000 points takes 1.7 seconds per intermediate frame, where the solution of the optimization problem on Mosek takes 0.86 seconds, and most of the remaining time is spent on the formulation of the problem by Yalmip. The timing was measured with an Intel i7 processor, clocked at 3.3 Ghz.

\section{Applications}

In this section we provide several applications and extensions of the method. These include morphing networks of curves with simple connectivity, morphing of curves with different turning numbers, convex combination of multiple curves, and regular homotopic morphing of $B$-spline curves using their control polygon.
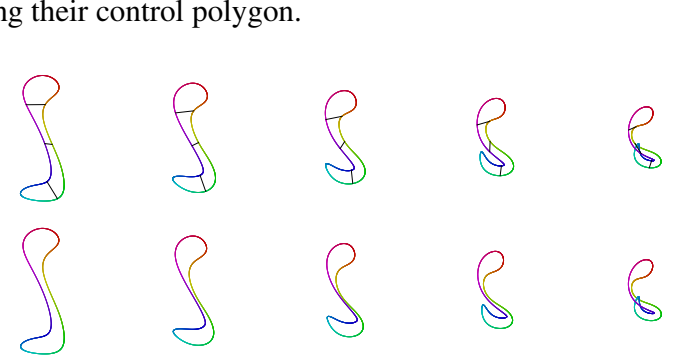

Figure 11: The addition of three diagonals (top) prevents the shrinking of the body of the snake which appears in our regular algorithm (bottom).

Network of Curves. We consider a generalization of the curve morphing problem to a certain class of polygonal curve networks. The motivation is to allow the user to specify diagonals in $\mathbf{c}^{0}, \mathbf{c}^{1}$ between two pairs of corresponding vertices to better control volume changes. Figure $11 \mathrm{com}$ pares our homotopic morphing method with our method of morphing networks of curves. The snake example shows how addition of diagonals can prevent the shrinking of the snake's body which appears in our homotopic morphing algorithm, which has direct control only on the distances between adjacent vertices. We note that in all other cases we encountered, shrinking did not occur and so it seems that in general our method does handles volume changes well, as illustrated in the examples in section 7.

To create a morph that is aware of the diagonals we model the problem using a network of polygonal curves: We assume the user added corresponding diagonals to the source and target, resulting in a collection of closed simple curves $\mathbf{c}^{k, m}, k=1,2, \ldots, K, m=0,1$, where every pair of curves $\mathbf{c}^{k_{1}, m}, \mathbf{c}^{k_{2}, m}$ either share a diagonal or are disjoint. The network of curves can be modeled using a single polygonal mesh (since both networks are equivalent) $M=(V, E, F)$, where $V$ is the index set of the vertices, $E$ the index set of the edges, and $F=\{k\}$ the index set of the curves. Since we only allow diagonals to be added, the dual graph of the mesh is a tree. It follows that we can perform our morphing method on each of the faces (curves) $\mathbf{c}^{k, m}, m=0,1$ separately, and then construct a solution to the morphing problem recursively; we begin by computing a regular homotopy for the root of the dual graph. We then preform a breadth-first search on the dual graph of the mesh, at every step constructing a regular homotopy for the current face and connecting the solution of the added face to the solution of the faces already connected, by applying scaling and rigid motion. While this approach is sub-optimal, it implies that the following generalization of (4.2) is still feasible:

$$
\begin{array}{ll}
\min _{L^{k, t}} \sum_{j, k}\left(\frac{L_{j}^{k, t}-\bar{L}_{j}^{k, t}}{\sqrt{\bar{L}_{j}^{k, t} L_{j}^{k, t}}}\right)^{2} \frac{1}{2}\left(L_{j}^{k, t}+\bar{L}_{j}^{k, t}\right) \\
\text { s.t. } \\
\quad L_{j}^{k, t} \geq 0 \\
\sum_{j} L_{j}^{k, t} e^{i \bar{\varphi}_{j}^{k, t}}=0 \quad \forall k \in F \\
\sum_{j, k} L_{j}^{k, t}=1 \\
L_{j}^{k, t}=L_{j^{\prime}}^{k^{\prime}, t} \\
\end{array}
$$

A realization of the mesh at time $t$ can be reconstructed from the solution $L^{k, t}, k=1 \ldots K$, and the linearly interpolated external angles of the curves via breadth first search as described above. This reconstruction is unique up to rotation and translation, and thus does not depend on the root chosen.

Curves with different turning numbers. We now consider the application of our algorithm to morphing polyg- 
onal curves $\mathbf{p}^{0}, \mathbf{p}^{1}$ with different turning numbers. We will give an overview of our results. The technical explanation appears in the paper's supplementary material.

The theory developed above for curves with identical turning number can be generalized to the case of curves with different turning numbers: Feasibility of (5.1) is guaranteed, under the additional assumption that $\tau\left(\mathbf{p}^{0}\right)$ and $\tau\left(\mathbf{p}^{1}\right)$ have the same sign. We thus assume w.l.o.g. that $0<\tau\left(\mathbf{p}^{0}\right)<$ $\tau\left(\mathbf{p}^{1}\right)$. Additionally, the number of flips through the morph will be exactly $\tau\left(\mathbf{p}^{1}\right)-\tau\left(\mathbf{p}^{0}\right)$, which is the minimal amount of flips possible.

Direct application of our algorithm (or the angle length method) will always cause flips in the first vertex only. We thus propose a strategy for choosing the correct first vertex based on minimizing work in the space of external angles. When $\tau\left(\mathbf{p}^{1}\right)-\tau\left(\mathbf{p}^{0}\right)=k>1$, more than one flip occurs. We explain how our method can be modified in this case so that the flips occur at $k$ different vertices, chosen such that work in the external angle space is minimized.

Figure 12 illustrates how applying the angle-length method directly causes pinching at the wrong vertex, and how this is fixed by our method for choosing the correct initial vertex.
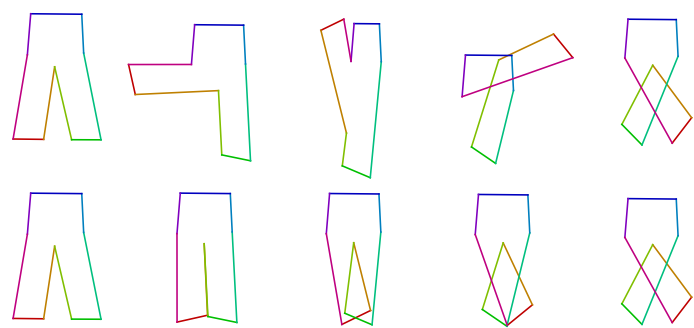

Figure 12: Morphing source and target curves whose turning numbers are one and two respectively. Using the anglelength method directly (top) gives unnatural results. Our automatic choice of the vertex at which flipping should occur (bottom) gives a natural morph.

Convex combination of multiple curves. Our method can be easily applied to the blending of more than two curves. The only difference being that the ideal curve $\overline{\mathbf{c}}^{t}$ is defined using more than two curves,

$$
\bar{\varphi}^{t}=\sum_{\ell} t_{\ell} \varphi^{\ell} \quad \bar{r}^{t}=\sum_{\ell} t_{\ell} r^{\ell}
$$

where $t_{\ell} \geq 0$, and $\sum_{\ell} t_{\ell}=1$. The projection $\mathbf{c}^{t}=\mathcal{P}\left(\overline{\mathbf{c}}^{t}\right)$ is performed as before. Figure 13 shows an example of blending the four curves at the corners of the square using different weights $t_{\ell}$ to produce a grid of intermediate curves.

Homotopic morphing of b-splines using the control polygon. We present an application of the monotone property

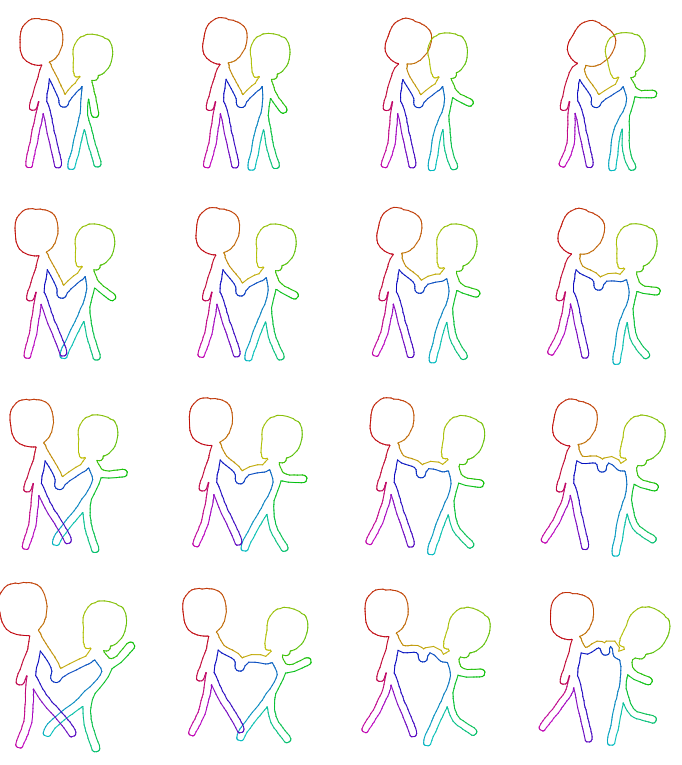

Figure 13: Morphing of the four curves at the corners of the rectangle.

from section 6 to the morphing of b-splines using their control polygon. We provide a sufficient condition which assures that morphing of $b$-splines via applying our morphing method to their control polygons, will give a regular homotopy. If the sufficient condition doesn't hold, it is possible to preform subdivisions on both control polygons curves until the sufficient condition is fulfilled.

Let us consider closed b-spline curves $\mathbf{c}:[0,1] \rightarrow \mathbb{R}^{2}$ which are piecewise polynomials of degree $D \geq 2$, defined by a sequence of increasing knots

$$
a_{-D}<\ldots<a_{0}=0<\ldots<a_{n}=1<\ldots<a_{n+D}
$$

and coefficients $\mathbf{p}_{1}, \ldots, \mathbf{p}_{n+D} \in \mathbb{R}^{2}$ so that

$$
\mathbf{c}(s)=\sum_{i=1}^{n+D} \mathbf{p}_{i} b_{i}(s)
$$

where $b_{i}$ are the $\mathrm{b}$-spline basis functions supported on $\left[a_{i-D-1}, a_{i}\right]$. The requirement that $\mathbf{c}$ are closed is equivalent to requiring

$$
\mathbf{p}_{i+n-1}=\mathbf{p}_{i}, \text { for } i=1, \ldots, D+1
$$

The control polygon of $\mathbf{c}$ consists of the first $n$ coefficients of $\mathbf{c}, \mathbf{p}=\left[\mathbf{p}_{1}, \ldots, \mathbf{p}_{n-1}, \mathbf{p}_{n}=\mathbf{p}_{1}\right]$. Assume we are given $\mathbf{c}^{0}$ and $\mathbf{c}^{1}$ of the same degree $D$, and defined over an identical knot vector by their control polygons $\mathbf{p}^{0}, \mathbf{p}^{1}$.

Lemma 8.1. If the control polygons $\mathbf{p}^{0}$ and $\mathbf{p}^{1}$ are regular and have the same non-zero turning number, and if all sub$\operatorname{arcs}\left[\mathbf{p}_{j}^{i}, \ldots, \mathbf{p}_{j+D}^{i}\right]$ are monotone, then the curve $\mathbf{c}^{t}$ obtained from the morphing of the control polygons is a well defined regular homotopy. 
An example of a b-spline curve with $D=3$ for which all sub-arcs with $D+1$ vertices are monotone can be seen in the inset.

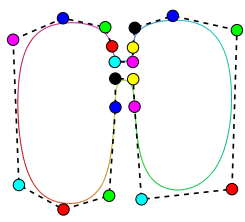

Proof. The fact that $\mathbf{c}^{t}$ are closed follows from construction. $\mathrm{c}^{t}$ are $C^{1}$, and therefore to show that they are regular it is sufficient to show that their derivative is always non zero. For $s \in\left(a_{i-1}, a_{i}\right)$, the derivative of $\mathbf{c}^{t}(s)=\sum_{i=j}^{j+D} \mathbf{p}_{i}^{t} b_{i}(s)$ is a positive conic combination of the edges $\mathbf{p}_{i+1}^{t}-\mathbf{p}_{i}^{t}$ for $i=j, \ldots j+D-1$ (e.g., page 67 in [PBP02]). Since $\left[\mathbf{p}_{j}^{t}, \ldots, \mathbf{p}_{j+D}^{t}\right]$ is monotone for $t=0,1$, it is monotone for all $t$ (see section 6), and this in turn implies that positive conic combinations of $\mathbf{p}_{i+1}^{t}-\mathbf{p}_{i}^{t}$ cannot be zero.

\section{Limitations}

Our morphing algorithm is not always applicable when the turning number of source and target curves is zero since the feasibility of the convex projection problem isn't guaranteed. The criterion for feasibility for this case is discussed in Appendix A. Similarly, the feasibility of our morphing scheme for curves with different turning numbers isn't guaranteed when the turning numbers of both curves don't have the same sign.

Our convex representation of regular closed curves fixes the angles to an ideal value, and closes the curve by allowing the lengths to change from their ideal value. This could be a slight disadvantage when an isometric morph is required, since such a morph requires fixed lengths throughout the morph. However we note that in practice our method seems to handle isometric morphing rather well as demonstrated in figure 4, 5,10,13.

\section{Conclusion}

We described an algorithm for morphing piecewise $C^{1}$ curves such that the morph is a regular homotopy, and the change in angles and lengths is 'as linear as possible'. The main novelty in this algorithm is our method for closing curves such that they are regular via a convex classification of the space of regular curves and the choice of a natural energy which is convex on this space and keeps the lengths of the curves away from zero.

We showed that the constructed morph has several attractive properties, including convexity preservation and the smoothness of the vertex trajectories.

We presented several applications and extensions of our method. We showed how our method can be extended to morphing of networks of curves with simple connectivity , and discussed morphing of curves with different turning numbers and convex combination of multiple curves. We also showed a sufficient condition for regular homotopic morphing of b-spline curves using their control polygons.
Acknowledgements We wish to thank Roi Poranne for advice and code sharing, Ben Antar for the source and target images in Figure 7 and the authors of [CWKBC13,SSHS14, BBA09] for code sharing. We also wish to thank the anonymous reviewers for their helpful comments.

This research was supported by the I-CORE program of the Israel PBC and ISF (Grant No. 4/11), the Israel Science Foundation (grant No. 1284/12), and the European Research Council (ERC Starting Grant, grant No.307754 - SurfComp)

\section{References}

[AA00] Andersen E. D., Andersen K. D.: The mosek interior point optimizer for linear programming: an implementation of the homogeneous algorithm. In High performance optimization. Springer, 2000, pp. 197-232. 9

[ACOL00] Alexa M., Cohen-Or D., Levin D.: As-rigid-aspossible shape interpolation. In Proceedings of the 27th annual conference on Computer graphics and interactive techniques (2000), ACM Press/Addison-Wesley Publishing Co., pp. 157164. 2

[ASS93] Aronov B., Seidel R., Souvaine D.: On compatible triangulations of simple polygons. Comput. Geom. Theory Appl. 3, 1 (June 1993), 27-35. 2

[BBA08] BAXTER W., BARLA P., ANJYO K.-I.: Rigid shape interpolation using normal equations. In Proceedings of the 6th international symposium on Non-photorealistic animation and rendering (2008), ACM, pp. 59-64. 2

[BBA09] BAXTER W., BARla P., ANJYO K.-I.: Compatible embedding for $2 \mathrm{~d}$ shape animation. Visualization and Computer Graphics, IEEE Transactions on 15, 5 (Sept 2009), 867-879. 11

[BBBK08] Bronstein A. M., Bronstein M. M., BRUCKSTEIN A. M., KimMEL R.: Analysis of two-dimensional nonrigid shapes. International Journal of Computer Vision 78, 1 (2008), 67-88. 8

[BV04] Boyd S., VAndenberghe L.: Convex Optimization. Cambridge University Press, New York, NY, USA, 2004. 13

[CPS13] Crane K., PINKALl U., SChrödER P.: Robust fairing via conformal curvature flow. ACM Trans. Graph. 32, 4 (July 2013), 61:1-61:10. 2, 3

[CWKBC13] CHEN R., Weber O., KEREN D., BEN-Chen M.: Planar shape interpolation with bounded distortion. ACM Trans. Graph. 32, 4 (July 2013), 108:1-108:12. 2, 8, 11

[GS01] GotSMAN C., SURAZHSKY V.: Guaranteed intersectionfree polygon morphing. Computers \& Graphics 25, 1 (2001), 67-75. 3

[HLZ04] Hu S.-M., LI C.-F., ZHANG H.: Actual morphing: a physics-based approach to blending. In Proceedings of the ninth ACM symposium on Solid modeling and applications (2004), Eurographics Association, pp. 309-314. 2

[IOD09] Iben H. N., O'Brien J. F., Demaine E. D.: Refolding planar polygons. Discrete \& Computational Geometry 41, 3 (2009), 444-460. 3

[KMP07] Kilian M., Mitra N. J., Pottmann H.: Geometric modeling in shape space. ACM Trans. Graph. 26, 3 (July 2007). 2

[KSMj04] Klassen E., Srivastava A., Mio W., Joshi S.: Analysis of planar shapes using geodesic paths on shape spaces. Pattern Analysis and Machine Intelligence, IEEE Transactions on 26, 3 (March 2004), 372-383. 2 
[LJ07] LiNG H., JACOBS D. W.: Shape classification using the inner-distance. IEEE Trans. Pattern Anal. Mach. Intell. 29, 2 (Feb. 2007), 286-299. 7

[Lof04] LofBerg J.: Yalmip: A toolbox for modeling and optimization in matlab. In Computer Aided Control Systems Design, 2004 IEEE International Symposium on (2004), IEEE, pp. 284289. 9

[MH47] Morse M., HeIns M.: Topological methods in the theory of functions of a complex variable. Bulletin of the American Mathematical Society 53 (01 1947), 1-14. 3

[MP06] Michor P M. D.: Riemannian geometries on spaces of plane curves. J. Eur. Math. Soc. 8 (2006), 1-48. 2

[MY91] Mehlhorn K., YAP C.-K.: Constructive whitneygraustein theorem: or how to untangle closed planar curves. SIAM J. Comput. 20, 4 (Aug. 1991), 603-621. 3

[PBP02] Prautzsch H., Boehm W., Paluszny M.: Bezier and B-Spline Techniques. Springer-Verlag New York, Inc., Secaucus, NJ, USA, 2002. 11

[Pin10] Pinkall U.: Mathematical visualization, lecture notes (unpublished), 2010. 3

[SE02] Surazhsky T., Elber G.: Metamorphosis of planar parametric curves via curvature interpolation. International Journal of Shape Modeling (2002), 201-216. 2

[SG92] Sederberg T. W., Greenwood E.: A physically based approach to $2 \mathrm{~d}$ shape blending. In Proceedings of the 19th Annual Conference on Computer Graphics and Interactive Techniques (New York, NY, USA, 1992), SIGGRAPH '92, ACM, pp. 25-34. 2

[SG95] SEDERBERG T. W., GreENWOOd E.: Shape blending of 2-d piecewise curves. In Mathematical Methods for Curves and Surfaces (1995), pp. 497-506. 2

[SGWM93] Sederberg T. W., Gao P., Wang G., Mu H.: 2$\mathrm{d}$ shape blending: an intrinsic solution to the vertex path problem. In Proceedings of the 20th Annual Conference on Computer Graphics and Interactive Techniques, SIGGRAPH 1993 (1993), pp. 15-18. 2, 4, 7, 8

[SR95] SHAPIRA M., RAPPOPORT A.: Shape blending using the star-skeleton representation. IEEE Comput. Graph. Appl. 15, 2 (Mar. 1995), 44-50. 2

[SSHS14] Saba M., SchneIder T., Hormann K., Scateni R.: Curvature-based blending of closed planar curves. Graph. Models 76, 5 (Sept. 2014), 263-272. 2, 7

[TvW02] TElEA A., VAN WIJK J. J.: An augmented fast marching method for computing skeletons and centerlines. In Proceedings of the Symposium on Data Visualisation 2002 (Airela-Ville, Switzerland, Switzerland, 2002), VISSYM '02, Eurographics Association, pp. 251-ff. 8

[WDAH10] Winkler T., Drieseberg J., Alexa M., HorMANN K.: Multi-scale geometry interpolation. In Computer graphics forum (2010), vol. 29, Wiley Online Library, pp. 309318. 2

[Whi37] WhitNeY H.: On regular closed curves in the plane. Compositio Mathematica 4 (1937), 276-284. 1, 3

\section{A. Proofs}

Proof of Lemma 5.3 Define

$$
A=\left\{\varphi: \operatorname{Coni}\left(e^{i \varphi}\right) \subseteq \operatorname{Coni}\left\{e^{i \varphi_{j}}: j \in\{1,2, \ldots, n-1\}\right\}\right\}
$$

We need to show $A=\mathbb{R}$. Note that
1. if $\alpha \in A$, also $\alpha+2 \pi \in A$. In particular it is sufficient to show $A$ contains the interval $\left[\varphi_{\min }, \varphi_{\min }+2 \pi\right]$.

2. if $\alpha, \beta \in A$ and $\alpha<\beta<\alpha+\pi$, then $\operatorname{Coni}\left\{e^{i \alpha}, e^{i \beta}\right\}$ contains all $r e^{i \delta}$ with $\delta \in[\alpha, \beta]$. Thus $[\alpha, \beta] \subseteq A$.

3. It follows that if $\varphi_{1}, \ldots \varphi_{n-1}$ satisfy $\left|\varphi_{j+1}-\varphi_{j}\right|<\pi$, then $\left[\varphi_{\min }, \varphi_{\max }\right] \subseteq A$. For $n-1=2$ this follows from the previous observation, and the general case can also be deduced from the previous observation by induction.

4. Now, if $\varphi_{\max }-\varphi_{\min } \geq 2 \pi$, then $\left[\varphi_{\min }, \varphi_{\min }+2 \pi\right] \subseteq A$ and we are done. Otherwise, $\varphi_{\max }<\varphi_{\min }+2 \pi<\varphi_{\max }+$ $\pi$ and so $\left[\varphi_{\max }, \varphi_{\min }+2 \pi\right] \subseteq A$, which again shows that $\left[\varphi_{\min }, \varphi_{\min }+2 \pi\right] \subseteq A$.

Feasibility, and zero turning number. We prove a slightly stronger version of Theorem 4.1.

Denote the set of non-singular points by $\Omega=[0,1] \backslash$ $\left\{\mathfrak{s}_{1}, \ldots, \mathfrak{s}_{n}\right\}$, and assume that $\varphi$, like $\varphi^{t}$ from Theorem 4.1, is piecewise continuous, and at singularities it satisfies

$$
\forall j,\left|\varphi_{+}\left(\mathfrak{s}_{j}\right)-\varphi_{-}\left(\mathfrak{s}_{j}\right)\right|<\pi
$$

Theorem A.1. There exists a $C^{1}$ function $r>0$ satisfying the closing constraint $\int r e^{i \varphi}=0$ iff

$$
\sup _{s_{1}, s_{2} \in \Omega} \varphi\left(s_{1}\right)-\varphi\left(s_{2}\right)>\pi
$$

Theorem 4.1 follows immediately, since when the turning number isn't zero, (A.1) holds for any $\varphi^{t}$ when $s_{1}, s_{2}$ are taken close to 0 and 1 . When the turning number is zero, we note that $\varphi^{0}$ and $\varphi^{1}$ fulfill (A.1) since the source and target curves are closed. As a result, for any given correspondence between $\mathbf{c}^{0}$ and $\mathbf{c}^{1}$, the correspondence can be 'corrected' so that there will be $s_{1}, s_{2}$ satisfying $\varphi^{t}\left(s_{1}\right)-\varphi^{t}\left(s_{2}\right)>\pi$ for $t=0,1$ and therefore for all $t \in[0,1]$. However, there may be correspondences in which the interpolation won't be feasible (unless corrected). For example, if $\varphi^{0}=-\varphi^{1}$, the optimization problem for $t=\frac{1}{2}$ won't be feasible.

\section{Proof. If (A.1) doesn't hold}

$$
\varphi_{\max }=\sup _{s \in \Omega} \varphi(s) ; \varphi_{\min }=\inf _{s \in \Omega} \varphi(s)
$$

satisfy $\varphi_{\max }-\varphi_{\min } \leq \pi$. Define $\varphi_{A}=\frac{\varphi_{\max }+\varphi_{\min }}{2}$. Note that for all $s \in \Omega,\left\langle e^{i \varphi_{A}}, e^{i \varphi(s)}\right\rangle \geq 0$ and there is some neighborhood in $\Omega$ such that for $s$ in the neighborhood the inner product is strictly positive. Thus if a positive $r$ with $\int_{0}^{1} r(s) e^{i \varphi(s)} d s=0$ existed, we would get a contradiction since

$$
\left\langle e^{i \varphi_{A}}, \int_{0}^{1} r(s) e^{i \varphi(s)} d s\right\rangle=\int_{0}^{1} r(s)\left\langle e^{i \varphi_{A}}, e^{i \varphi(s)}\right\rangle d s>0
$$

In the other direction, if (A.1) does hold, we can choose a sequence $s_{1}, \ldots, s_{n} \in \Omega$ such that $\varphi\left(s_{j}\right)$ satisfy the conditions of Lemma 5.3. Since the conditions are open, we can choose 
smooth positive $b_{j}$ supported in a small enough neighborhood of $s_{j}$, such that the neighborhood doesn't contain singular points, and

$$
\int_{0}^{1} b_{j}(s) e^{i \varphi(s)} d s=e^{i \varphi_{j}}
$$

where $\varphi_{j}$ are close enough to $\varphi\left(s_{j}\right)$ so that they also fulfill the conditions of Lemma 5.3. Therefore there is a positive solution $L_{j}>0$ to the equation

$$
\sum_{j=1}^{n} L_{j} e^{i \varphi_{j}}=-\int_{0}^{1} e^{i \varphi(s)} d s
$$

using (A.2) we obtain

$$
\int_{0}^{1}\left(\sum_{j=1}^{n} L_{j} b_{j}(s)+1\right) e^{i \varphi(s)} d s=0
$$

Proof of Lemma 5.2. The unique minimizer $L^{t} \in \mathbb{R}^{n}$ of (5.1) satisfies the KKT conditions: Denote the objective function from (5.1) by $f$ and write the constraint $\sum L_{j}^{t} e^{i \varphi_{j}}=$ 0 as $A(t) L^{t}=0$ where

$$
A(t)=\left(e^{i \varphi_{1}^{t}}, \ldots, e^{i \varphi_{n}^{t}}\right) \in \mathbb{R}^{2 \times n}
$$

Since the constraints $L_{j}^{t} \geq 0$ aren't active, the KKT conditions at time $t$ can be written as:

$$
\exists \mathrm{v}^{t} \in \mathbb{R}^{3}, L^{t} \in \mathbb{R}_{+}^{n} \text { solving the equations }
$$

$$
\begin{aligned}
& G_{1}(t, L, v) \equiv \nabla f(L)+\left[v_{1} v_{2}\right] A(t)+v_{3} \overrightarrow{1}^{T}=0 \\
& G_{2}(t, L) \equiv\left(L^{T} A^{T}(t), \overrightarrow{1}^{T} L-1\right)=(0,0)
\end{aligned}
$$

Denoting the hessian of $f$ by $H_{f}$ and

$$
B(t)=\left[\begin{array}{c}
A(t) \\
\overrightarrow{1}^{T}
\end{array}\right]
$$

the differential of the function $G(t, L, v)=$ $\left(G_{1}(t, L, v), G_{2}(t, L)\right)^{T}$ is given by

$$
d G(t, L, v)=\left[\begin{array}{ccc}
\frac{\partial G_{1}}{\partial t} & H_{f} & B(t)^{T} \\
\frac{\partial G_{2}}{\partial t} & B(t) & 0
\end{array}\right]
$$

We will show that the sub-matrix of partial derivatives according to $(L, v)$,

$$
\left[\begin{array}{cc}
H_{f} & B(t)^{T} \\
B(t) & 0
\end{array}\right]
$$

is non-singular for all $t$, and therefore by the implicit function theorem for every $t$ there are unique $\left(L^{t}, v^{t}\right)$ satisfying $G\left(t, L^{t}, v^{t}\right)=0$, and $\left(L^{t}, v^{t}\right)$ is a smooth function of $t$. This will prove the Lemma since $L^{t}$ is exactly the unique minimizer of the optimization problem at time $t$.

(c) 2015 The Author(s)

Computer Graphics Forum (c) 2015 The Eurographics Association and John Wiley \& Sons Ltd.
We note that $H_{f}$ is positive semi-definite since $f$ is strictly convex. According to ( [BV04] page 557), to prove nonsingularity it is sufficient to show that $B(t)$ is full rank. To see this, note that $A(t)$ is full rank, since for a regular closed curve $e^{i \varphi_{j}^{t}}$ cannot all be linearly dependent. It remains to show that there is no solution to the equation $A^{T}(t) \lambda=\overrightarrow{1}$. Indeed, assume such a solution exists, and choose $L>0$ satisfying $A(t) L=0$. This causes a contradiction since

$$
0=L^{T} A(t)^{T} \lambda=L^{T} \overrightarrow{1}>0
$$

